\title{
Rituximab-induced neutropenia in a patient with inflammatory myopathy and systemic sclerosis overlap disease
}

\author{
Qasim Akram ${ }^{1}$, Mark Roberts ${ }^{2}$, Chester Oddis ${ }^{3}$, Arianne Herrick ${ }^{1}$, Hector Chinoy ${ }^{1}$ \\ ${ }^{1}$ Rheumatology Department, Salford Royal Foundation Trust, Manchester, United Kingdom \\ ${ }^{2}$ Neurology Department, Salford Royal Foundation Trust, Manchester, United Kingdom \\ ${ }^{3}$ Division of Rheumatology and Clinical Immunology, University of Pittsburgh, Pennsylvania, USA
}

\begin{abstract}
Rituximab (RTX) is a monoclonal chimeric antibody directed against the CD20 antigen of B lymphocytes. Late onset neutropenia ( $L O N$ ) is a recognised complication of rituximab usually occurring 4 weeks after the last dose and is reported in both haematological and rheumatological conditions. However, it has never been described in a patient with myositis and systemic sclerosis overlap disease. We describe a case of LON in a 54-year-old man who was diagnosed with myositis and then systemic sclerosis overlap disease. It resolved within 7 days, and the patient did not suffer neutropenic sepsis or any other complications. We propose similar mechanisms for LON as described in other conditions and routine blood monitoring in such patients.
\end{abstract}

Key words: rituximab, myositis, systemic sclerosis, neutropenia.

\section{Introduction}

Rituximab, a monoclonal chimeric antibody directed against the CD20 antigen of B lymphocytes, is used in the treatment of haematological malignancies, rheumatoid arthritis and ANCA-associated vasculitis. There is evidence for use of rituximab in systemic lupus erythematosus (SLE) and idiopathic inflammatory myopathy (IIM).

A recognised complication of rituximab in lymphoma is late onset neutropenia (LON), which may be defined as grade 3 (neutrophil count $0.5-1.0 \times 10^{6}$ ) or grade $4\left(<0.5 \times 10^{6}\right)$ in the absence of other causes, occurring at least 4 weeks after rituximab [1]. Data regarding LON in rheumatic autoimmune conditions are sparse [1]. To date, there have been no reports of rituximab-induced neutropenia in idiopathic inflammatory myopathy (IIM) [2]. We describe a case of rituximab-induced neutropenia in a patient with refractory $1 \mathrm{MM}$ /systemic sclerosis overlap.

\section{Case report}

A 54-year-old man presented with a 10-month history of chest pain and painful hands, shoulders and thighs. He described difficulty rising from a chair and lifting his arms above his head. Cardio-respiratory and abdominal systems were unremarkable, with no sclerodermatous features initially evident. There was quadriceps wasting with associated proximal weakness on Kendall scale manual muscle testing [3]: hip flexion $7 / 10$, knee extension 8/10, and shoulder abduction 9/10 bilaterally (MMT24 248/260). Serum creatine kinase (CK) was $10.813 \mu / /(<190 \mu / l)$, cardiac troponin T (cTnT) $169 \mathrm{ng} / \mathrm{l}$ (<14 ng/l), ANA 1/1000 speckled pattern. Myositis specific/associated antibody testing using serum immunoprecipitation was negative, although anti-Ro52 was weakly positive on immunoblot. Anti-topoisomerase, anti-RNA polymerase III and anti-HMG CoA reductase antibody tests were also negative. The initial ECG and echocardiogram were unremarkable. Electromyography showed 


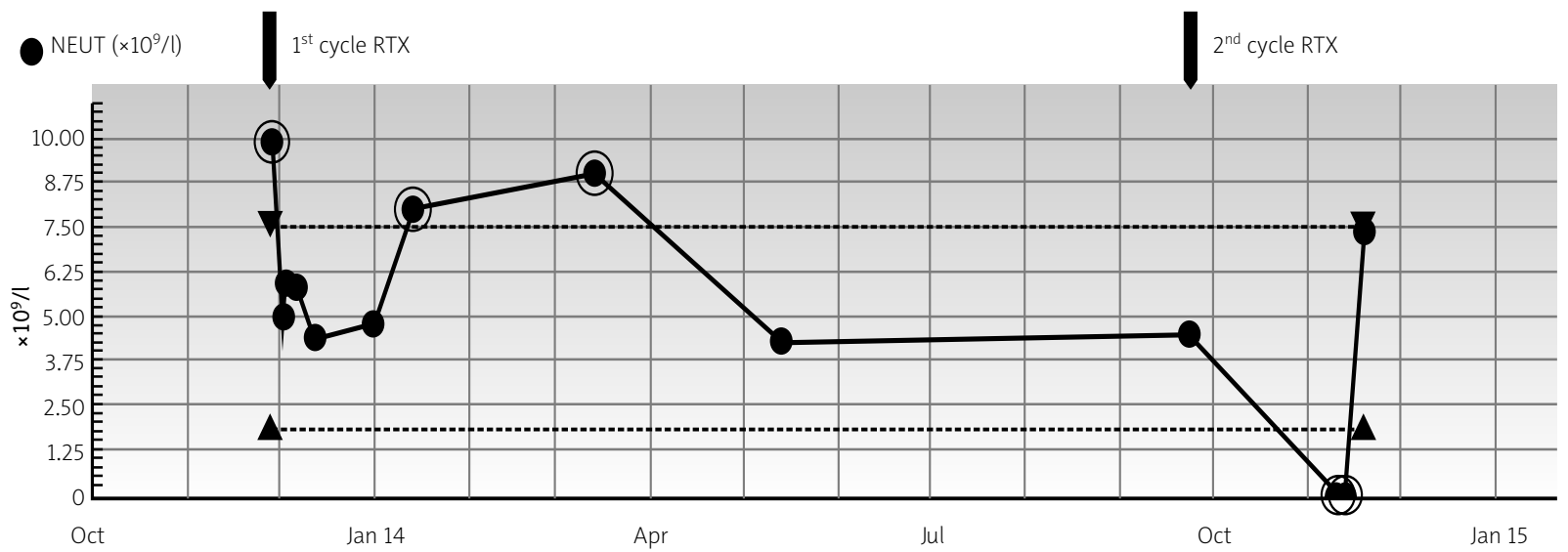

Fig. 1. Diagram showing trend of neutrophils following rituximab (RTX) administration.

lower limb myopathic unit potentials. MR thigh imaging showed muscle oedema on short tau inversion recovery sequences with no T1 fatty muscle replacement. A trapezius muscle biopsy showed necrotic/regenerative activity, occasional non-necrotic fibres with invasion by inflammatory cells, and widespread strong HLA-1 upregulation. An initial diagnosis of IIM with additional features of necrotizing myopathy was made.

He was commenced on $60 \mathrm{mg}$ prednisolone and after one month due to clinical non-response received six cycles of cyclophosphamide as per CYCLOPS [4]. He improved clinically but the $\mathrm{CK}$ remained raised $(4,500 \mu / \mathrm{l})$. Cyclophosphamide was discontinued as the patient felt markedly unwell after treatment. Steroids were further weaned as IIM symptoms had improved. Methotrexate was not tolerated; mycophenolate mofetil was then introduced but stopped one year later after symptoms of abdominal pain and diarrhoea thought to be due to pseudo-intestinal obstruction. He then received three cycles of IV immunoglobulin, which dramatically improved his symptoms.

Four months later the patient was admitted due to dizziness and loss of consciousness, with shortness of breath, poor exercise tolerance, orthopnoea and paroxysmal nocturnal dyspnoea. ECG showed runs of non-sustained ventricular tachycardia, an echocardiogram showed left ventricular systolic dysfunction with severe impairment, and cardiac MR showed dilated biventricular size and poor global systolic function. The patient also reported widespread skin tightness with worsening Raynaud's phenomenon but no digital ulcers. A modified Rodnan score of 24 confirmed the diagnosis of IIM/systemic sclerosis overlap with inflammatory cardiac disease activity and damage. An emergency cardiac defibrillator was implanted.

For treatment escalation, he received $2 \times 1 \mathrm{~g}$ IV rituximab infusions as per the standard rheumatoid arthritis protocol [5]. Four months later, there was a significant improvement; MMT24 252/260, CK 282 H/l, cTnT 79 ng/l. A second cycle of $1 \mathrm{~g}$ rituximab was commenced six months after the first cycle.

A full blood count one month after the $2^{\text {nd }}$ cycle revealed leucopenia $\left(2.5 \times 10^{9}\right)$ and profound neutropenia $\left(0 \times 10^{9}\right)$, confirmed on repeat testing, with normal haemoglobin and platelets. The patient remained well with no infectious symptoms and was on no medications that could cause leucopenia. Haematology consultation advised repeating the full blood count one week later and admission to hospital should he develop pyrexia or systemic features. A full blood count one week later showed resolution of both the neutropenia and leucopenia (Fig. 1), and during this period he remained well.

\section{Discussion}

Rituximab-induced neutropenia in autoimmune disease has been reported at a similar frequency seen in haematological malignancies, between $3 \%$ and $27 \%$, after approximately 5 months of rituximab administration $[1,6,7]$. In the landmark Rituximab in Myositis Trial, no cases of neutropenia were recorded [2]. Oddis et al. [2] reported seven episodes of leucopenia in 200 cases, ranging from 2.7 to $3.9 \times 10^{9}$ (personal correspondence).

The underlying mechanism of rituximab-induced LON remains poorly understood. Theories have included the development of novel anti-neutrophil antibodies and proliferation of rituximab-induced T cell large granulocyte lymphocytes leading to apoptosis of mature neutrophils [7]. Perturbation of stromal derived factor 1 level during $B$ cell recovery may prevent neutrophil egress from bone marrow [6, 7]. However, Tesfa et al. [7] have presented flow cytometry data indicating that LON may occur at any stage of B cell recovery. Patients with LON also seem to have a longer and excessive duration of $\mathrm{B}$ cell depletion, generally resolving without require- 
ment for colony stimulating factor. The clinical picture observed in our case is similar to that seen in haematological malignancies and other autoimmune diseases, where studies quote resolution of the neutropenia after a mean of 6.5 days [6].

This is the first case of neutropenia related to rituximab in a patient with IIM spectrum disorder.

The authors declare no conflict of interest.

\section{References}

1. Breuer G, Ehrenfeld M, Rosner I, et al. Late onset neutropenia following rituximab for rheumatic conditions. Clin Rheumatol 2014; 33: 1337-1340.

2. Oddis CV, Reed AM, Aggarwal R, et al.; RIM Study Group. Rituximab in the Treatment of Refractory Adult and Juvenile Dermatomyositis and Adult Polymyositis A Randomized, PlaceboPhase Trial. Arthritis Rheum 2013; 65: 314-324.

3. Rider LG, Giannini EH, Harris-Love M, et al.; International Myositis Assessment and Clinical Studies Group. Defining Clinical Improvement in Adult and Juvenile Myositis. J Rheumatol 2003; 30: 603-617.

4. De Groot K, Muhler M, Reinhold-Keller E, et al. Randomised controlled trial of daily oral versus pulsed cyclophosphamide for induction of remission in ANCA associated systemic vasculitis (abstract). Kidney Blood Pressure Res 2005; 28: 195.

5. Emery P, Rigby WF, Combe B, et al. Efficacy and safety of rituximab (RTX) as first-line biologic therapy in patients with active rheumatoid arthritis (RA): results of a phase III randomized controlled study (SERENE). Arthritis Rheum 2008; 58: S302.

6. Besada E, Koldingsnes W, Nossent J. Characteristics of late onset neutropenia in rheumatology patients: a case review analysis from a single centre. QJ Med 2012; 105: 545-560.

7. Tesfa D, Ajeganova S, Hagglund H, et al. Late onset neutropenia following rituximab therapy in rheumatic diseases: association with B lymphocyte depletion and infections. Arthritis Rheum 2011; 63: 2209-2214. 